\title{
TAK1 regulates endothelial cell necroptosis and tumor metastasis
}

\author{
Lida Yang ${ }^{1}$ - Sayali Joseph ${ }^{1} \cdot$ Tianliang Sun ${ }^{1,3} \cdot$ Julia Hoffmann $^{1,4} \cdot$ Sophia Thevissen $^{1} \cdot$ Stefan Offermanns ${ }^{1,2}$. \\ Boris Strilic ${ }^{1}$
}

Received: 13 September 2018 / Revised: 18 December 2018 / Accepted: 21 December 2018 / Published online: 25 January 2019

(c) ADMC Associazione Differenziamento e Morte Cellulare 2019

\begin{abstract}
Formation of metastases is the major cause of death in patients diagnosed with cancer. It is a complex multistep process, including tumor cell migration, intravasation, survival in the circulation, and extravasation. Previously it was shown that tumor cell-induced endothelial necroptosis promotes tumor cell extravasation and metastasis. Here, we identified endothelial TGF- $\beta$-activated kinase 1 (TAK1) as a critical regulator of endothelial necroptosis and metastasis. Human and murine endothelial cells lacking TAK1 exhibit higher levels of necroptosis both in vitro and in vivo, and mice with endothelial cellspecific loss of TAK1 are more prone to form metastases. Endothelial RIPK3, a key component of the necroptotic machinery, was upregulated in mice with endothelial TAK1-deficiency, and endothelial knockout of RIPK3 reverted the effects of TAK1-deficiency. Moreover, altered expression levels of TAK1 and RIPK3 in pulmonary endothelial cells of mice bearing primary tumors correlated with increased endothelial necroptosis and metastasis. Together, our data suggest an important protective role for endothelial TAK1 in tumor progression by keeping endothelial necroptosis in check.
\end{abstract}

\section{Introduction}

Metastasis is a process during which individual tumor cells (TCs) spread mostly through the circulatory system to colonize distant organs. The first step in the metastatic

These authors contributed equally: Stefan Offermanns, Boris Strilic

Edited by H. Ichijo

Supplementary information The online version of this article (https:// doi.org/10.1038/s41418-018-0271-8) contains supplementary material, which is available to authorized users.

Stefan Offermanns

Stefan.Offermanns@mpi-bn.mpg.de

$\triangle$ Boris Strilic

Boris.Strilic@mpi-bn.mpg.de

1 Max Planck Institute for Heart and Lung Research, Department of Pharmacology, Ludwigstr. 43, 61231 Bad Nauheim, Germany

2 J.W. Goethe University Frankfurt, Center for Molecular Medicine, Theodor-Stern-Kai 7, 60590 Frankfurt, Germany

3 Present address: Novartis Institutes for Biomedical Research, Novartis Pharma AG, WSJ360.4.20 Novartis Campus, 4056 Basel, Switzerland

4 Present address: Charité Universitätsmedizin Berlin, Institute of Pathology, Charitéplatz 1, 10177 Berlin, Germany cascade is the escape of individual TCs from the primary tumor and their subsequent invasion of surrounding tissue. Once in the circulation, the metastatic potential of TCs largely depends on a rapid and efficient way to escape from the blood stream by passing the endothelial barrier [1-3]. However, the mechanisms underlying TC extravasation are still incompletely understood. It is believed that endothelial permeability must be elevated for TCs to extravasate, which for example can be achieved through opening of interendothelial junctions via various mechanisms [4-7]. More recently, it was found that endothelial necroptosis promotes transendothelial migration of TCs and metastasis [8].

Necroptosis is a programmed form of necrosis, which can be induced by the activation of various receptor systems including death receptors of the TNF receptor superfamily. Recruitment of signaling molecules to death receptors and formation of membrane-associated or cytosolic complexes, depending on cellular conditions and other signals, drives transcription of genes that prevent cell death and sustain inflammation or induce cell death in the form of apoptosis or necroptosis, respectively [9]. Execution of necroptosis depends on the assembly of the necrosome, a complex consisting of receptor-interacting serine/threonineprotein kinase 1 (RIPK1) and RIPK3, which activates the pseudokinase mixed lineage kinase domain-like (MLKL) eventually leading to plasma membrane rupture $[9,10]$. Even though the molecular regulation of the transitions 
between cell survival and the cell death pathways are incompletely understood, transforming growth factor $\beta$ (TGF- $\beta$ )-activated kinase 1 (TAK1) seems to play an important role in regulating these decisions $[11,12]$.

Here, we show that loss of TAK1 in endothelial cells (ECs) sensitizes them to increased basal necroptosis that facilitates TC transendothelial migration and metastasis. Endothelial cells lacking TAK1 express higher levels of RIPK3 suggesting that TAK1 functions to keep necroptosispromoting factors at low levels. Interestingly, lung endothelial cells of mice harboring primary tumors show altered gene expression levels of TAK1 and RIPK3 that correlate with increased endothelial necroptosis and metastasis.

\section{Methods and materials}

\section{Cells}

Human umbilical vein endothelial cells (HUVEC) and medium were from Lonza. MDA-MB-231-GFP cells were from AntiCancer. B16F10, LLC1, MDA-MB-468, MCF-7, and HEK293T cells were from ATCC. All cells were incubated at $37{ }^{\circ} \mathrm{C}$ and $5 \% \mathrm{CO}_{2}$. HUVECs were cultured in growth factor-supplemented medium (EGM-2), and passages $<\mathrm{P} 7$ were used for all experiments. All other cell lines were cultured in either RPMI or DMEM supplemented with $10 \%$ FBS, penicillin/streptomycin (100 units/ml) and glutamine $(2 \mathrm{mM})$. Cells were tested negative for mycoplasma contamination before experiments.

\section{Cell death assays and analysis}

For co-culture experiments, HUVECs $\left(1.5 \times 10^{4}\right.$ at seeding in $100 \mu \mathrm{l}$ ) were cultured for $24 \mathrm{~h}$ in 96-well plates in EGM-2 medium. To induce cell death, $1.5 \times 10^{3}$ MDA-MB-231GFP TCs were added to the HUVEC monolayer and cultured overnight. For knockdown experiments, $1.5 \times 10^{4}$ HUVECs were transfected using Lipofectamine RNAiMAX (Life Technologies) with different sets of siRNAs (Sigma) and cultured on 96-well plates. Knockdown efficiencies were determined by western blotting upon lysis with Laemmli buffer or by quantitative RT-PCR (LightCycler480, Roche). For experiments with TCconditioned medium, HUVEC monolayers grown to confluency were cultured for $24 \mathrm{~h}$ with conditioned medium obtained from MDA-MB-231-GFP, MDA-MB-468, and MCF-7 TCs that were cultured in EGM-2 for $24 \mathrm{~h}$. For all conditions, EthD-III (1.6 $\mu \mathrm{M}$, Biotium) and Hoechst 33342 $(2 \mu \mathrm{M}$, Thermo Scientific) were added shortly before automated image acquisition in an atmosphere-controlled chamber $\left(37^{\circ} \mathrm{C}, 5 \% \mathrm{CO}_{2}\right)$ using an Olympus IX81 microscope or before overnight culture. All images were analyzed in ImageJ (National Institutes of Health). Morphological criteria for discriminating necroptotic cells from apoptotic or living cells were defined as before [8]. Unless stated otherwise, each experiment was performed at least three times with a minimum of six wells per condition and four independent images acquired per well.

\section{Identification of potential molecules that promote TC-induced EC death}

The screen to identify potential molecules that mediate TCinduced EC death was performed in a 96-well format. HUVECs were transfected with siRNAs targeting mRNAs of individual genes (Table 1) resulting in knockdown efficiencies $>75 \%$. Forty-eight hours after gene silencing, $2 \times 10^{3}$ MDA-MB-231-GFP TCs were seeded on confluent monolayers of HUVECs in the presence of $100 \mu \mathrm{M} z-V A D-$ fmk. After overnight co-culture, the number of dead ECs was determined as described above. The screen was performed in five independent rounds with duplicates in each round. The ratio of EC necroptosis for each condition was defined as the effect of each siRNA to alter endothelial necroptosis compared to control HUVECs (scrambled siRNA). Background cell death in HUVECs transfected with scrambled siRNA and cultured without TCs was determined for each plate individually and subtracted from each value. For values $>1$, gene knockdown resulted in an increase in TC-induced endothelial necroptosis; for values $=1$, gene knockdown resulted in no change; for values $<1$, gene knockdown resulted in a decrease in TC-induced endothelial necroptosis.

\section{Transwell assays}

Assays were performed as described previously [4]. Briefly, $8 \times 10^{3}$ HUVECs were transfected with different sets of siRNA and cultured on 96-transwell plates with polyester membranes of $8 \mu \mathrm{m}$ pore size (Corning) with daily medium changes until reaching confluency. For TC transmigration experiments, the medium of the upper compartment was removed and $7.5 \times 10^{3}$ GFP-expressing TCs were added in $50 \mu \mathrm{l}$ endothelial culture medium. Transmigrated TCs on the lower side of the filter were imaged (Zeiss Axio Observer Z1 or Olympus IX81) and quantified with ImageJ. Each experiment was performed at least three times with a minimum of six wells per condition. For permeability assays, the medium of the upper compartment was removed and FITC-Dextran $(70 \mathrm{kD}, 1.0 \mathrm{mg} / \mathrm{mL}$, Invitrogen) was added in $100 \mu \mathrm{l}$ endothelial culture medium. Permeability was determined by measuring the fluorescence at $538 \mathrm{~nm}$ (485 $\mathrm{nm}$ excitation) using a FlexStation 3 fluorescence reader (Molecular Devices) that was emitted from $10 \mu \mathrm{L}$ medium aliquots taken from the bottom chambers and further diluted in phosphate-buffered saline (PBS). 


\section{Mouse experiments}

Control C57BL/6J animals were purchased from Charles River. TAK1 $1^{\text {loxP/loxP }}$ animals [13], RIPK3 ${ }^{\text {loxP/loxP }}$ animals [8] or $\mathrm{MLKL}^{-1-}$ animals [8] were crossed to Tamoxifeninducible Tie2-CreER ${ }^{\mathrm{T2}}$ animals [14] to obtain Tie2-

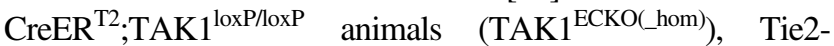
$\mathrm{CreER}^{\mathrm{T} 2} ; \mathrm{TAK}^{\text {loxP/+}}$ animals (TAK1 ${ }^{\mathrm{ECKO}}{ }^{\mathrm{Ehet}}$ ), Tie2-CreER ${ }^{\mathrm{T} 2}$; TAK1 $1^{\text {loxPlloxP}} ;$ RIPK $3^{\text {loxP/loxP }}\left(\mathrm{TAK}^{\mathrm{ECKO}} ;{ }^{\mathrm{EIPK}} 3^{\mathrm{ECKO}}\right.$ ) or Tie2$\mathrm{CreER}^{\mathrm{T} 2} ; \mathrm{TAK}^{\text {loxPlloxP}}{ }^{\mathrm{MLKL}^{-/-}} \quad$ (TAK1 $\left.^{\mathrm{ECKO}} ; \mathrm{MLKL}^{-1-}\right)$. Mice were maintained under specific pathogen free conditions. Quantification of in vivo permeability was performed using the Miles assay. Briefly, $11 \mathrm{~d}$ after induction of the knockout by Tamoxifen $(1 \mathrm{mg}$ per mouse in $50 \mu \mathrm{l}$ Miglyol i.p. for 5 consecutive days), mice received a $100 \mu \mathrm{l}$ tail vein injection of $0.5 \%$ Evans blue dye in PBS. After 30 min mice were killed, and extravasated blue dye was eluted from the lungs with formamide at $56^{\circ} \mathrm{C}$ and measured by spectrometry at $620 \mathrm{~nm}$. For tumor-related experiments $50 \mu \mathrm{l}$ PBS containing $5 \times 10^{4}$ TCs (B16F10 melanoma or LLC1 lung carcinoma cells) were injected i.v. For evaluation of TC-induced EC death $50 \mu \mathrm{l} \mathrm{EthD-III}(300 \mu \mathrm{M}$ in PBS $)$ were injected i.v. $6 \mathrm{~h}$ after injection of TCs, and 10 min thereafter animals were killed and perfused with PBS and 4\% paraformaldehyde and directly processed for immunohistochemical analysis. For evaluation of the number of extravasated TCs, carboxyfluorescein diacetate succinimidyl ester-labeled B16 TCs were injected i.v. and $6 \mathrm{~h}$ later nonperfused lungs were isolated and fixed in $4 \%$ paraformaldehyde. Stained cryosections were analyzed in XYZ views on a Leica SP5 or SP8 confocal microscope. The number of EthD-III-positive ECs was determined by manual counting of EthD-III/ERGor EthD-III/CD31-double-positive cells on central areas of a minimum of four random longitudinal sections of the left lobe and the superior and middle right lobe of each lung. Numbers of EthD-III-positive ECs from each section of one lung were averaged (average per mouse), and these values were again averaged to obtain a mean value \pm s.e.m. from one experiment. For quantification of extravasated TCs, cryosections were analyzed by two criteria: TCs directly surrounded by CD31 staining (i.e., blood vessel) and with a noninvasive phenotype (i.e., round cell shape) were scored as intravascular, while cells outside blood vessels with an invasive phenotype (i.e., irregular cell shape with protrusions) were scored as extravascular. Lung metastases were analyzed $12 \mathrm{~d}$ after i.v. injection without EthD-III treatment. The mean number of lung metastases per experiment was determined by averaging the individual values of metastases from each lung resulting in a mean value \pm s.d. from one experiment. For primary tumor models, $10^{6} \mathrm{~B} 16$ or LLC1 TCs in $50 \mu \mathrm{l}$ PBS were injected subcutaneously into the shaved flank of mice, tumor sizes were measured three times per week as described previously [15], and lung metastases were evaluated either macroscopically or by analyzing every tenth section after whole-lung sectioning and H\&E staining using standard procedures. To study the effects of primary tumors on lung EC necroptosis and metastasis formation, $10^{6}$ B16 or LLC1 TCs in $50 \mu$ PBS were injected subcutaneously into the shaved flank of mice, and $12 \mathrm{~d}$ later $5 \times 10^{4}$ TCs (B16 or LLC1) in $100 \mu \mathrm{l}$ were injected intravenously. The first group was treated $6 \mathrm{~h}$ thereafter with EthD-III and lungs of mice were analyzed for EC necroptosis. Primary tumors from mice of the second group were surgically removed $24 \mathrm{~h}$ after i.v. injection of TCs and analysis of tumor metastasis was performed another $11 \mathrm{~d}$ later. Mice treated with PBS served as controls. For all experiments, animal groups were sex-matched, and mice were 8-16 weeks of age. A minimum of three animals per group was used, and mice were grouped randomly. Protocols were performed according to institutional and national guidelines. All experimental animal procedures were approved by the Hessian Regional Board.

\section{Generation of RIPK3-overexpressing cells}

To generate HUVEC cells expressing full-length RIPK3, a modified lentiviral $\mathrm{pLVX}$ expression vector containing the sequence of human RIPK3 with a puromycin resistance marker for selection was transfected into HEK293T cells along with envelope plasmid pMD2.G and packaging plasmid psPAX2. After $48 \mathrm{~h}$ of incubation, lentiviral supernatant was collected and filtered through a $0.45 \mu \mathrm{m}$ low-protein binding durapore membrane (Millex). HUVECs at approximately $60 \%$ confluency were transduced with lentivirus $(50 \%$ filtered lentiviral supernatant and 50\% fresh EGM-2 medium) plus $8 \mu \mathrm{g} / \mathrm{mL}$ Polybrene (Sigma) for $12 \mathrm{~h}$, followed by complete medium change. To eliminate nontransduced cells, puromycin (Sigma) was added freshly to the medium at a final concentration of $0.6 \mu \mathrm{g} / \mathrm{mL} 24 \mathrm{~h}$ later, and the puromycin-containing medium was replaced every $2 \mathrm{~d}$. After $4 \mathrm{~d}$, the transduced cells were used for further analyses. pLVX-tdTomato-N1 served as control and was a gift from Johnny Kim (Max Planck Institute for Heart and Lung Research, Germany).

\section{Expression analyses}

HUVEC from individual wells of a 6- or 96-well plate were harvested $\left(1.5 \times 10^{5}\right.$ or $5 \times 10^{3}$ cells at seeding $)$ and RNA was isolated and transcribed into cDNA according to the manufacturers' protocols (Qiagen, Roche). For quantitative RT-PCR using the Roche LightCycler480 Probes Master System, 30 ng cDNA per reaction were used. Intron-spanning primers were designed with the online tool provided by Roche, and only primer pairs from the top three results were chosen. Relative expression levels were obtained by normalizing to GAPDH. 
For mouse lung endothelial cell isolation, mice were treated as described above and lungs were minced and incubated in digestion buffer $(1.2 \mathrm{U} / \mathrm{ml}$ Dispase, $2 \mathrm{mg} / \mathrm{ml}$ Collagenase II, $0.04 \mathrm{mg} / \mathrm{ml}$ Elastase, and $5 \mathrm{U} / \mathrm{ml}$ DNaseI in DMEM medium) for $10-20 \mathrm{~min}$ at $37^{\circ} \mathrm{C}$ while shaking. After filtration the cells were washed in PBS containing $0.5 \%$ bovine serum albumin. Cells were incubated with anti-CD45-APC (Thermo Fisher Scientific) and anti-CD31-PE (Thermo Fisher Scientific) for 20 min at $4{ }^{\circ} \mathrm{C}$ and CD45-negative/CD31-positive endothelial cells were sorted with FACS Aria III (BD).

\section{Other materials}

Media and supplements were from Life Technologies. The following reagents were used: tamoxifen (Sigma), z-VADfmk (APExBIO), calcein-AM (AAT Bioquest), and DAPI (Life Technologies). The following antibodies were used for western blot analyses: anti-TAK1 (sc-7162, Santa Cruz), anti-cIAP2 (ALX-803-341-C100, Enzo), anti-MLKL (107538, GeneTex), anti-RIPK3 (NBP2-24588, Novus biologicals), anti- $\alpha$-tubulin (T9026, Sigma), and antiGAPDH (2118L, Cell signaling). The following antibodies were used for immunohistochemistry: anti-CD31 (550274, BD Biosciences), anti-cleaved caspase-3 (9661S, Cell Signaling), and anti-ERG (ab110639, Abcam).

\section{Statistical analysis}

Trial experiments or experiments done previously were used to determine sample size with adequate statistical power. In vitro experiments were not randomized and the investigators were not blinded to them, whereas the in vivo experiments were randomized and investigators were blinded. Samples were excluded in cases where RNA/cDNA quality or tissue quality after processing was poor (below commonly accepted standards). Animals were excluded from experiments if they showed any signs of sickness. Data represent biological replicates. In all studies, comparison of mean values was conducted with unpaired, two-tailed Student's $t$ test or one-way ANOVA with Bonferroni's post hoc test. In all analyses, statistical significance was determined at the $5 \%$ level $(P<0.05)$. Depicted are mean values \pm s.d. or \pm s.e.m. as indicated in the figure legends. Statistical analysis was performed with Prism5 or Prism6 (GraphPad) or Excel software (Microsoft).

\section{Results}

\section{Absence of TAK1 promotes endothelial necroptosis and TC metastasis}

A previous study showed that endothelial necroptosis facilitates TC extravasation and metastasis [8]. To identify
Table 1 Sequences of siRNAs (Sigma, human)

\begin{tabular}{|c|c|}
\hline Gene name & Target sequence $\left(5^{\prime}->3^{\prime}\right)$ \\
\hline CASP1 & GAGAUCCUUCUGUAAAGGU \\
\hline CASP8 & GATTTATCATCACCTCAAA \\
\hline CFLAR (FLIP) & GGGAUGUUGCUAUAGAUGU \\
\hline CHUK (IKK $\alpha)$ & AGGGCAGCAAUGUUAAGUC \\
\hline CIAP1 & GUGAGUUCUUGAUACGAAU \\
\hline CIAP2 & GAUUGUGCUCCUUCUUUAA \\
\hline CYLD & GUCAUUACAAUUCUUGUUA \\
\hline FADD & CUCAAGCUGCGUUUAUUUAA \\
\hline IKBKB $(\mathrm{IKK} \beta)$ & GGACAUUGUUGUUAGCGAA \\
\hline IKBKG (Nemo) & CUCUUCCAAGAAUACGACA \\
\hline MAP3K7 (TAK1) \#1 & GUGUUUACAGUGUUCCCAA \\
\hline MAP3K7 (TAK1) \#2 & CCUGAAACCACCAAACUUA \\
\hline MAP3K7 (TAK1) \#3 & GGUUGCUGAGGCCCUGUAA \\
\hline MLKL \#1 & CCAACATCCTGCGTATATT \\
\hline MLKL \#2 & GCTAAGAAGAGATAATGAA \\
\hline MYD88 & GCAUUGAGGAGGAUUGCCA \\
\hline PGAM5 & CCGAUAUCAUCAGCCGGCA \\
\hline RBCK1 (HOIL-1) & GUGCCUACCUCUAUCUGCU \\
\hline RIPK3 \#1 & GCGATATCCAGGGAGGTCA \\
\hline RIPK3 \#2 & GACAACAACTACTTGACTA \\
\hline RNF31 (HOIP) & GAAACUACCUCAACACCCU \\
\hline SHARPIN & CCUGGAAACUUGACGGAGA \\
\hline TAB1 & CGGCUAUGAUGGCAACCGA \\
\hline TAB2 & GGAAUUUCUGGUCUACGCA \\
\hline TICAM1 (TRIF) & GCACUGAACGCAGCCUACU \\
\hline TNFAIP3 (A20) & GGAUGUUACCAGGACAUUU \\
\hline TRADD & CCAAUGGCGGCCUGGCCUA \\
\hline TRAF1 & GAACCCAUCUGUCGCUCUU \\
\hline TRAF2 & GCCUUCAGGCCCGACGUGA \\
\hline TRAF3 & GUCAAGAGAGCAUCGUUAA \\
\hline TRAF4 & GCACUAAGGAGUUCGUCUU \\
\hline TRAF5 & GAGAAGUCCUCAACUUAUA \\
\hline TRAF6 & CAUUCUGAAGGAUUGUCCA \\
\hline TRAF7 & CACAUCAACGCGCGGCUGA \\
\hline XIAP & GAUUAAAGUCCUUUCAGAA \\
\hline ZBP1 (DAI) & CCUCAAUUAUUUACCAGCA \\
\hline
\end{tabular}

potential regulators of endothelial necroptosis we performed an siRNA-mediated screen in HUVECs. Among the 33 chosen candidates, all of which participate in necroptotic signaling pathways, molecules such as Caspase-8, MLKL, RIPK3, and RIPK1 were previously shown to affect TCinduced endothelial necroptosis and thus served as controls [8]. Of the remaining ones, we found that knockdown of $M A P 3 K 7$, the gene coding for TAK1 (transforming growth factor beta (TGF- $\beta$ )-activating kinase 1$)$, resulted in increased necroptosis in HUVECs when cultured in the presence of MDA-MB-231 TCs (Fig. 1a), and experiments 
with two independent siRNAs confirmed this (Fig. 1b and Supplementary Figure S1a). Moreover, we found that TAK1 silencing induced endothelial necroptosis independent of TC stimulation (Fig. 1b). Since the degree of EC necroptosis correlates with the ability of TCs to pass an endothelial cell layer [8], we examined whether
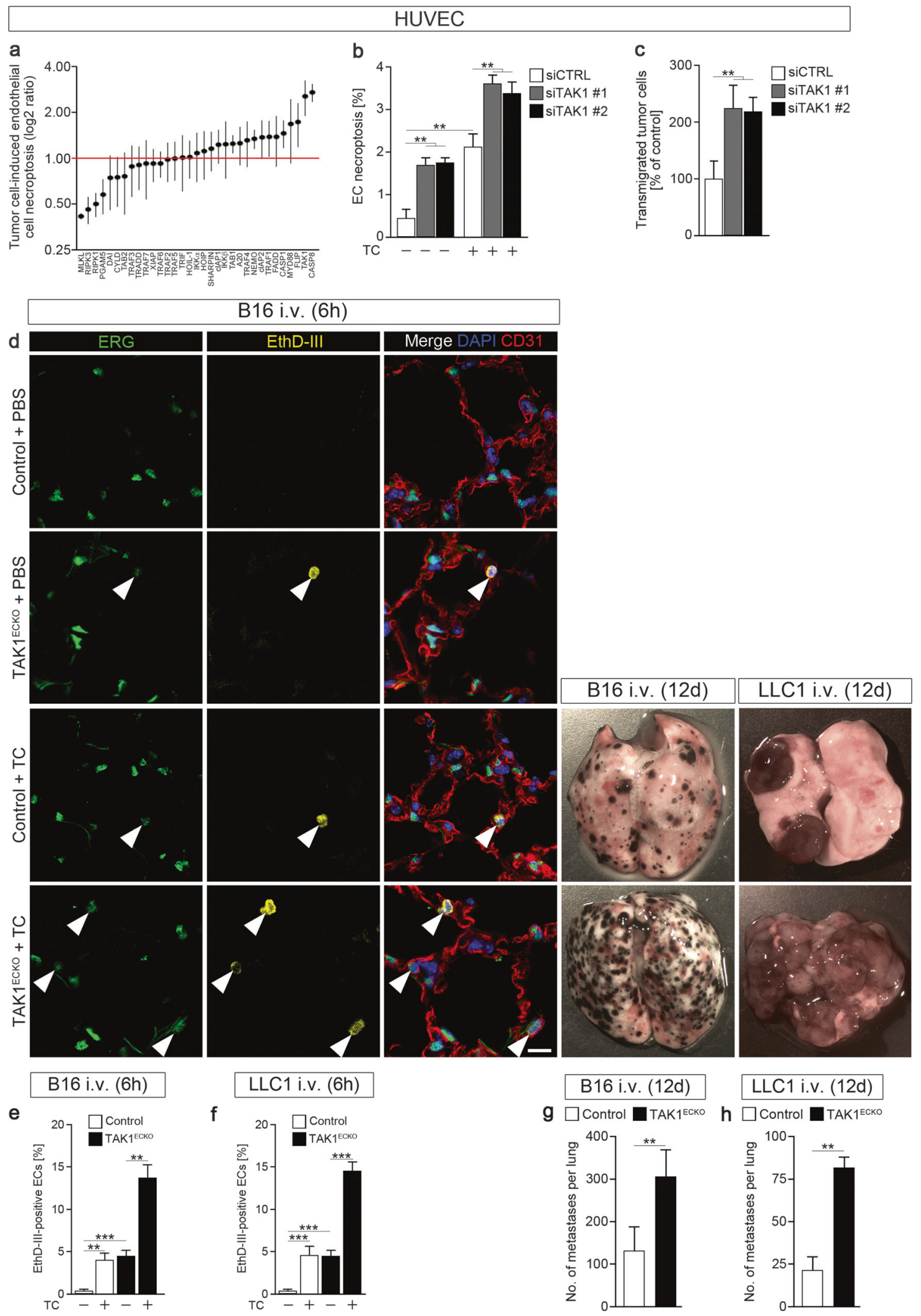
Fig. 1 Absence of endothelial TAK1 promotes endothelial necroptosis and tumor cell metastasis. a Effects of endothelial siRNA knockdowns on MDA-MB-231 TC-induced EC necroptosis (HUVECs) compared to scrambled siRNA. b, c Knockdown of TAK1 in ECs and quantification of EC necroptosis in the absence (-) or presence (+) of TCs (b) and transmigrated TCs over a monolayer of HUVECs (c). d Confocal images of lung sections $6 \mathrm{~h}$ after i.v. injection of PBS or B16 TCs into Cre-negative littermates (control) or tamoxifen-inducible ECspecific TAK1 knockout mice $\left(\mathrm{TAK} 1^{\mathrm{ECKO}}\right.$ ) and stained for the indicated markers. Arrowheads, EthD-III-positive cells. Bar length: $20 \mu \mathrm{m}$. Images of whole lungs $12 \mathrm{~d}$ after i.v. injection of B16 or LLC1 TCs are shown on right. e, f Quantification of pulmonary EthD-III-positive ECs $6 \mathrm{~h}$ after i.v. injection of B16 (e) or LLC1 (f) TCs into Crenegative control animals or TAK1 ${ }^{\mathrm{ECKO}}$ mice. $\mathbf{g}, \mathbf{h}$ Quantification of tumor metastases in lungs $12 \mathrm{~d}$ after i.v. injection of B16 (g) or LLC1 (h) TCs. Shown are representative data of three independent experiments with mean values \pm s.e.m. $(\mathbf{a}, \mathbf{b}, \mathbf{e}, \mathbf{f})$ or \pm s.d. $(\mathbf{c}, \mathbf{g}, \mathbf{h})$ from biological sextuplicates $(n=6)(\mathbf{a}-\mathbf{c})$ or from $n=4-6$ animals per condition $(\mathbf{e}-\mathbf{h}) . * * P<0.01 ; * * * P<0.001$. One-way ANOVA and Bonferroni's post hoc test (a-c, e, f) or unpaired, two-tailed Student's $t$ test $(\mathbf{g}, \mathbf{h})$. Mice treated with PBS served as controls

TAK1-deficiency in ECs facilitates TC transendothelial migration. After endothelial knockdown of TAK1, we found increased numbers of transmigrated TCs as compared to control conditions (Fig. 1c), while the permeability for fluorescein isothiocyanate (FITC)-dextran was unchanged in ECs lacking TAK1 under basal conditions (Supplementary Figure S1b).

We next tested whether absence of TAK1 in endothelial cells in vivo also leads to increased basal necroptosis and whether this has an effect on TC metastasis. While no necroptosis was observed in Cre-negative control littermates (Control), mice with tamoxifen-inducible endothelial cell-specific knockout of TAK1 (Tie2-CreER ${ }^{\mathrm{T} 2} ; \mathrm{TAK}^{\mathrm{f} / \mathrm{fl}}$ ) showed increased numbers of pulmonary EthD-III-positive cells $\left(\mathrm{TAK} 1^{\mathrm{ECKO}}\right.$ ) (Fig. 1d, e). These necroptotic cells costained with ERG, a nuclear marker for endothelial cells (Fig. 1d). Similar to the in vitro data, the degree of endothelial permeability in the lungs of these animals was not changed (Supplementary Figure S1c), and no change in endothelial apoptosis was observed (Supplementary Figure S1d). Six hours after intravenous injection of syngeneic B16F10 melanoma (B16) or Lewis Lung Carcinoma (LLC1) TCs into TAK1 $1^{\mathrm{ECKO}}$ mice $\left(\mathrm{TAK} 1^{\mathrm{ECKO}}+\mathrm{TC}\right)$, the degree of endothelial necroptosis was further increased as compared to $\mathrm{TAK} 1^{\mathrm{ECKO}}$ mice alone (TAK1 $\left.{ }^{\mathrm{ECKO}}+\mathrm{PBS}\right)$ or as compared to control littermates treated with TCs (Control + TC) (Fig. 1d-f). Importantly, in correlation with the increase in necroptosis, $\mathrm{TAK} 1^{\mathrm{ECKO}}$ mice were more prone to develop metastases $12 \mathrm{~d}$ after intravenous injection of TCs (Fig. 1d, g, h). These mice also developed more lung metastases derived from primary tumors upon subcutaneous implantation although the size of the primary tumors was significantly smaller (Supplementary Figure 1e-j). Moreover, heterozygous $\mathrm{TAK} 1^{\mathrm{ECKO}}$ mice $\left(\mathrm{TAK} 1^{\mathrm{ECKO} \_ \text {het }}\right.$ ) showed intermediate levels of metastasis when compared to wild-type control littermates and homozygous knockout mice (TAK1 $1^{\text {ECKO_hom }}$ ) (Supplementary Figure S1k, 1). Together, these data show that absence of endothelial TAK1 sensitizes ECs towards necroptosis and promotes metastasis.

\section{Absence of TAK1 sensitizes ECs towards increased necroptosis via RIPK3}

Since it is known that TAK1 has a prosurvival role in mediating activation of the NF- $\kappa$ B pathway which has a number of target genes, many of which block cell death [16], we asked whether knockdown of TAK1 in ECs resulted in altered gene expression of molecules that may explain increased necroptosis. While mRNA levels of most of the analyzed genes were unchanged, we found reduced expression levels of the ubiquitin E3 ligase cIAP2 (BIRC3) in TAK1-deficient HUVECs (Fig. 2 and Supplementary Figure S2a). Even though cIAP2 has well-known prosurvival functions by ubiquitinating RIPK1 and thus to promote cell death when absent or inactive [17, 18], knockdown of cIAP2 alone in ECs by siRNA did not induce necroptosis and had no effect on TC transendothelial migration (Supplementary Figure S2b, c).

Besides changes in cIAP2 expression, we found increased expression levels of RIPK3 upon knockdown of TAK1 (Fig. 2). In line with the notion that RIPK3 is a key mediator of necroptotic cell death signaling [9], we found increased numbers of necroptotic ECs when overexpressing RIPK3 in HUVECs (Fig. 3a and Supplementary Figure S3a). Furthermore, increased necroptosis upon RIPK3 overexpression resulted in more TCs migrating over an endothelial layer (Fig. 3b). In both cases, knockdown of MLKL, a substrate of RIPK3 required for the execution of necroptosis [9], reverted these effects (Fig. 3a, b and Supplementary Figure S3b).

To test whether increased TC-induced EC necroptosis and TC transendothelial migration in the absence of TAK1 can be normalized by knockdown of RIPK3, we performed double knockdown experiments by generating ECs lacking both TAK1 and RIPK3 and compared their ability to mediate TC transendothelial migration to ECs lacking only TAK1. Indeed, additional knockdown of RIPK3 blocked EC necroptosis induced by TAK1-deficiency (Fig. 3c and Supplementary Figure S3c), and reduced the number of transmigrated TCs (Fig. 3d). To further test this in vivo, we generated double knockout mice lacking both TAK1 and RIPK3 specifically in endothelial cells $\left(\mathrm{TAK} 1{ }^{\mathrm{ECKO}}\right.$;RIP$\mathrm{K} 3^{\mathrm{ECKO}}$ ). Similar to the in vitro findings, additional knockout of RIPK3 in mice lacking TAK1 resulted in less EC necroptosis and metastases of B16 or LLC1 TCs (Fig. 3e-g and Supplementary Figure S3d, e). Moreover, 


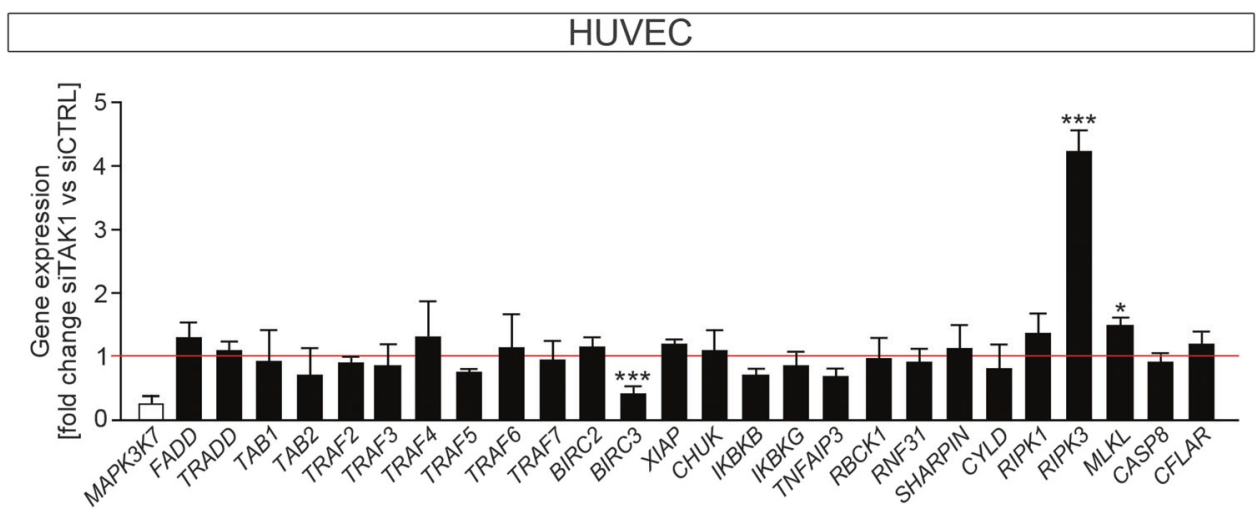

Fig. 2 Expression levels of cell death-related molecules are changed upon TAK1 knockdown in endothelial cells. Analysis of changes in gene-expression levels upon siRNA-mediated knockdown of TAK1 in HUVECs (siTAK1) as compared to cells treated with scrambled

additional knockout of MLKL in TAK1 ${ }^{\mathrm{ECKO}}$ animals also resulted in reduced numbers of metastases (Supplementary Figure S3f, g). Together, these data suggest that the increased metastasis seen after induction of endothelial TAK1-deficiency is due to increased necroptosis.

\section{Primary tumors influence the premetastatic niche by inducing necroptosis}

It is well established that the vasculature at premetastatic sites is remodeled by the primary tumor through various, poorly understood mechanisms which can create a favorable environment for incoming TCs [19]. In ECs cultured in the presence of TC-conditioned medium we found reduced levels of TAK1 and increased levels of RIPK3 (Fig. 4a and Supplementary Figure S4a), and the degree of necroptosis was increased (Fig. 4b). Moreover, we observed the same changes in gene expression when analyzing pulmonary ECs of mice with subcutaneously implanted B16 or LLC1 primary tumors (Fig. 4c). To assess whether primary tumors also have the ability to induce necroptosis in ECs at future sites of metastasis, we analyzed the lungs of wild-type animals $12 \mathrm{~d}$ after subcutaneous injection of B16 or LLC1 TCs, a time when metastasis from these tumors is not yet detectable $[20,21]$. We found that the degree of necroptotic lung ECs in these animals (TC s.c.) was increased as compared to control mice (PBS) and comparable to mice that received TCs via intravenous injection for $6 \mathrm{~h}$ (TC i.v.). When tumor-bearing mice were additionally injected intravenously with TCs (TC s.c. + TC i.v.), we observed additional effects on pulmonary EC necroptosis (Fig. 4d, e and Supplementary Figure S4b, c and data not shown). These effects were comparable between B16 or LLC1 TCs injected into B16 tumor-bearing mice, or B16 or LLC1 TCs injected into LLC1 tumor-bearing mice (data not shown).
siRNA (siCTRL). Shown are representative data of three independent experiments with mean values \pm s.d. from biological duplicates. $* P<$ $0.05 ; * * * P<0.001$. One-way ANOVA and Bonferroni's post hoc test

This prompted us to further test whether necroptosis induced in lung ECs by a primary tumor also facilitates TC extravasation and metastasis. To this end, we subcutaneously implanted primary tumors and allowed them to grow for $12 \mathrm{~d}$ to induce premetastatic alterations, followed by intravenous injection of TCs to mimic the metastatic phase [21]. Six hours after intravenous injection of TCs into tumor-bearing mice we found more extravascular TCs in the lungs as compared to mice without primary tumors (Fig. 4f). To evaluate the effects on tumor metastasis, primary tumors were surgically excised $24 \mathrm{~h}$ after intravenous injection to exclude contribution of metastasizing TCs originating from the primary tumor, and lungs were analyzed $11 \mathrm{~d}$ thereafter (Supplementary Figure S4b). Since the immune system in a host with a progressive tumor rejects the same TCs at distant sites, known as concomitant tumor immunity [22, 23], we injected B16 TCs into mice bearing primary tumors derived from LLC1 cells. In these mice, the number of B16 lung metastases was significantly increased as compared to control mice without a primary tumor (Fig. 4d, g), and these effects were reverted in mice lacking endothelial RIPK3 (Supplementary Figure S4d, e). Taken together, these data show that primary tumors affect expression levels of TAK1 and RIPK3 and are able to induce metastasis-promoting necroptosis in ECs at metastatic sites.

\section{Discussion}

It is well established that future metastatic sites could be selectively and actively modified by poorly defined factors coming from the primary tumor before metastatic spread has even occurred [19]. As a result, the formation of such premetastatic niches provides a favorable environment for the dissemination of TCs by facilitating adhesion, 

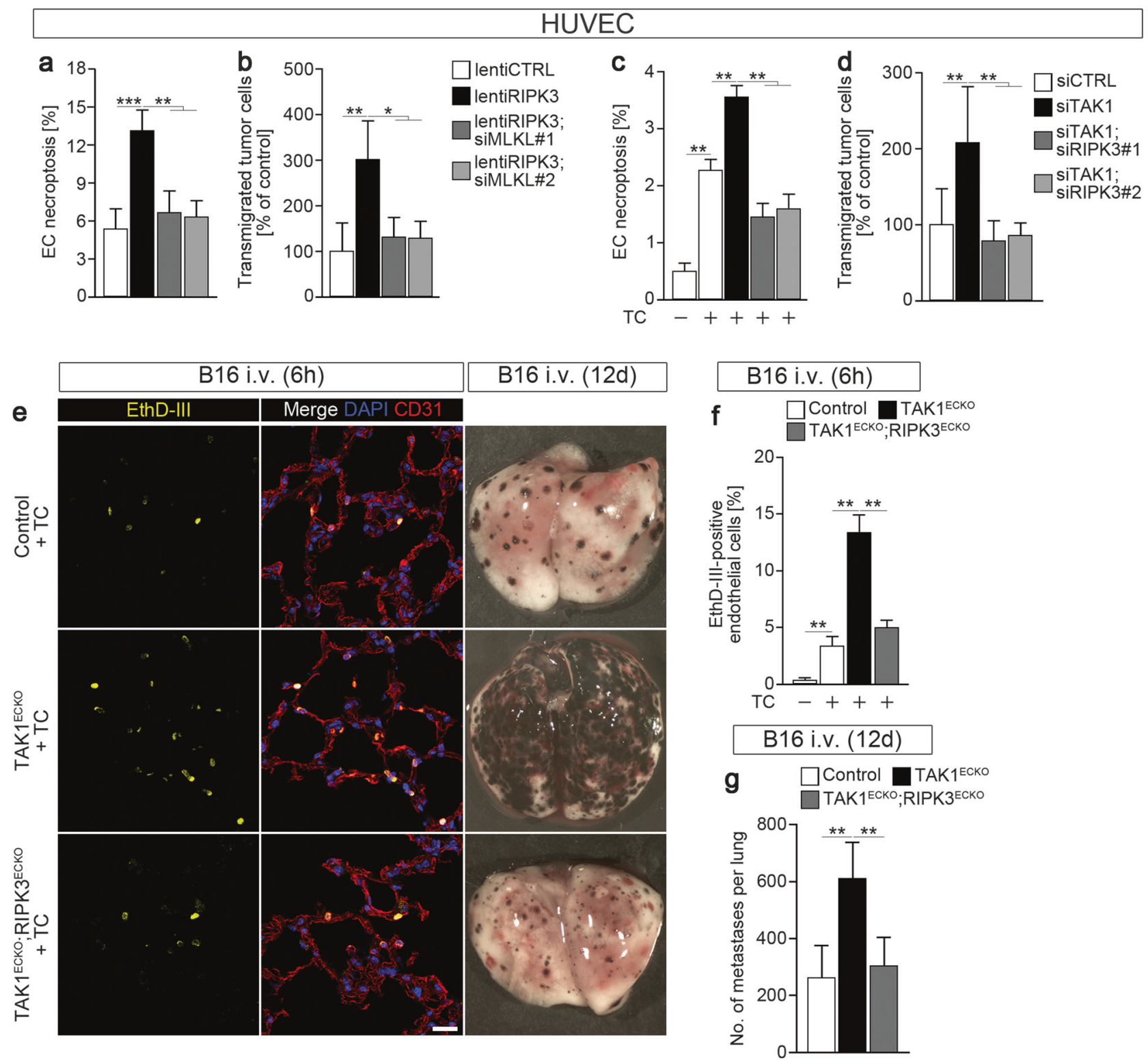

Fig. 3 Absence of TAK1 sensitizes endothelial cells toward increased necroptosis via RIPK3. a-d HUVECs were treated with lentiviruses to overexpress RIPK3 (lentiRIPK3), siRNA against TAK1 (siTAK1), MLKL (siMLKL), or RIPK3 (siRIPK3). Quantification of MDA-MB231 TC-induced necroptosis in HUVECs $(\mathbf{a}, \mathbf{c})$ and degree of transmigrated MDA-MB-231 TCs over a HUVEC monolayer (b, d) as indicated. e-g B16 TCs were i.v. injected into Cre-negative littermates (control), TAK $1^{\mathrm{ECKO}}$ or $\mathrm{TAK} 1^{\mathrm{ECKO}} ; \mathrm{RIPK} 3^{\mathrm{ECKO}}$ mice, and after $6 \mathrm{~h}$

extravasation, migration, and/or proliferation of metastasizing TCs. Consistent with the concept that tumor-derived factors induce a premetastatic niche characterized by specific changes in endothelial cells [19, 24], we found that primary tumors alter gene expression in mouse lung ECs. In these mice, endothelial TAK1 levels were reduced and RIPK3 expression was increased, which furthermore coincided with increased numbers of necroptotic ECs, facilitated TC extravasation and metastasis. The factors responsible for lungs were imaged as indicated (e) and analyzed for pulmonary EthDIII-positive ECs (f) or lungs were analyzed after $12 \mathrm{~d}$ for lung metastases (g). Bar length: $20 \mu \mathrm{m}$. Shown are representative data of three independent experiments with mean values \pm s.e.m. $(\mathbf{a}, \mathbf{c}, \mathbf{f})$ or \pm s.d. (b, d, g) from biological sextuplicates $(n=6)(\mathbf{a}-\mathbf{d})$ or from $n=4$ 6 animals per condition (f, g). $* P<0.05 ; * * P<0.01 ; * * * P<0.001$. One-way ANOVA and Bonferroni's post hoc test. Mice treated with PBS served as controls

the development of such premetastatic niches are believed to be directly secreted into the circulation by cells of the primary tumor or to be transported via tumor-shed extracellular vesicles or exosomes and include inflammatory cytokines and chemokines, hormones, proangiogenic factors, extracellular matrix components, metabolites and lipids, as well as genetic material, including DNA, mRNA, and miRNA $[19,24]$. Among these, miRNAs were shown to alter TAK1 expression levels [25-28], to regulate 
necroptosis [29] and to affect vascular integrity and to promote metastasis [30]. Furthermore, high serum levels of TAK1-repressing miRNAs were shown to correlate with poor prognosis in cancer patients [31,32]. It is, however, likely that besides miRNAs, several other factors directly or indirectly and possibly in a synergistic manner affect ECs within the premetastatic niche $[21,33,34]$ to regulate gene expression and to induce metastasis-promoting necroptosis in endothelial cells.

TAK1 is known to act as key regulator of cell survival and cell death signaling, which is in line with our observation that loss of TAK1 increased endothelial cell death.
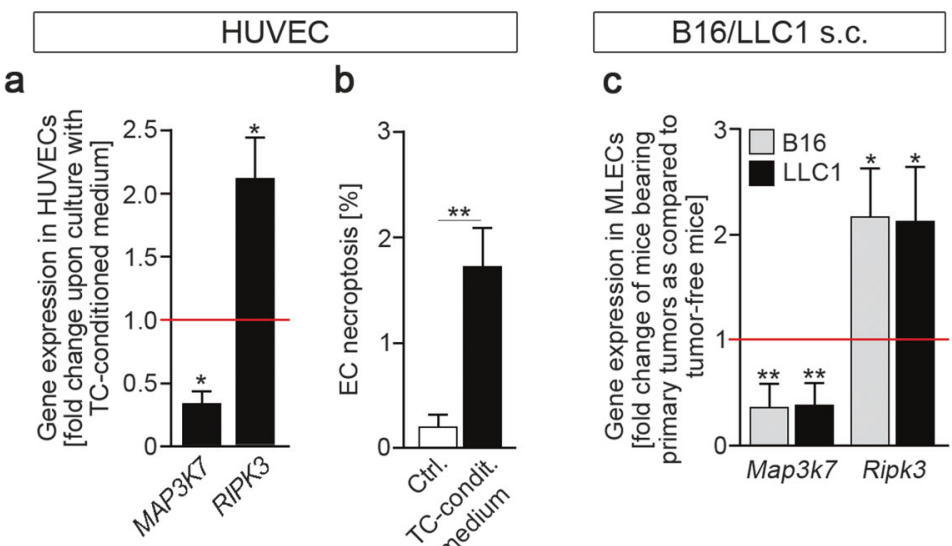

b
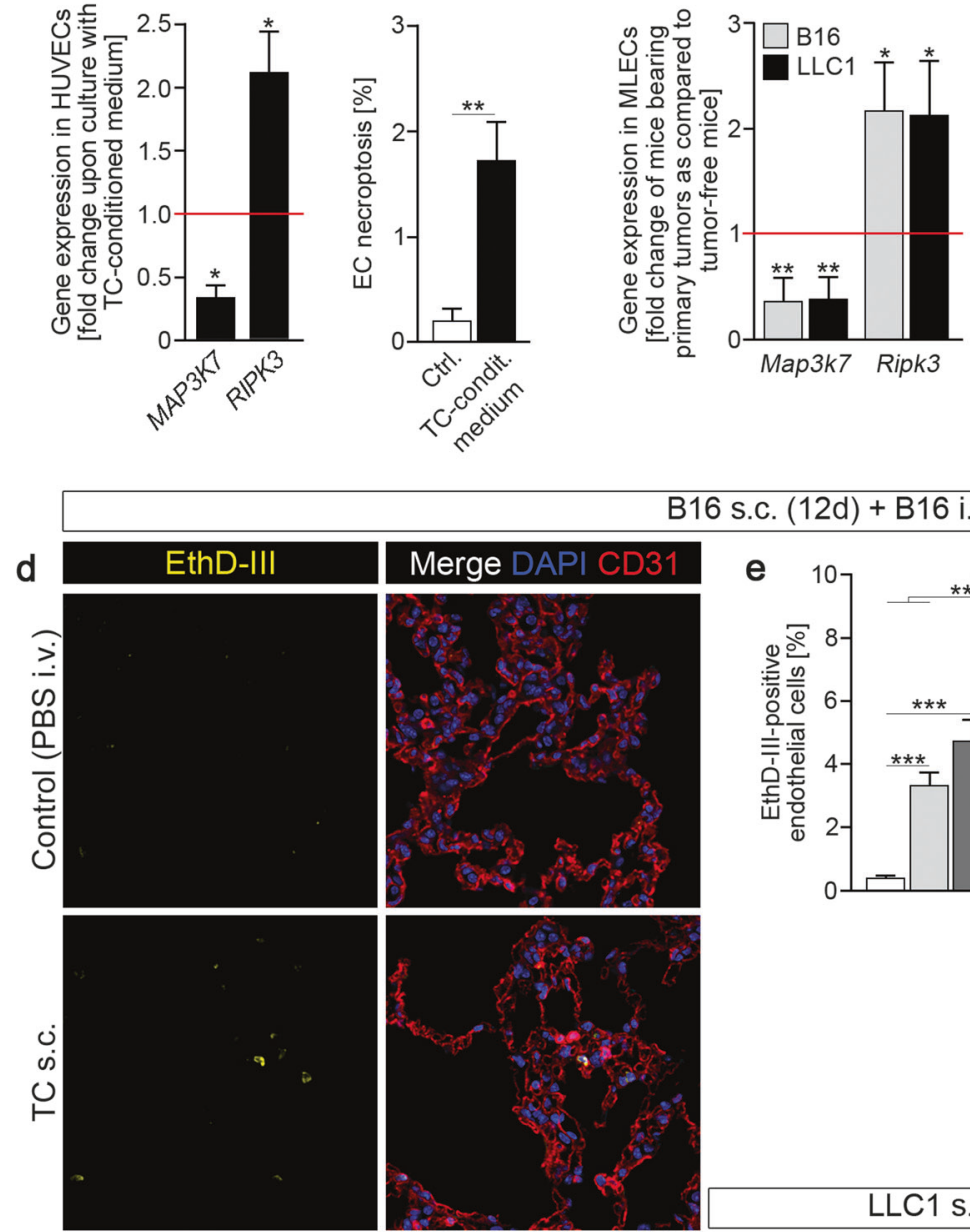

B16 s.c. (12d) + B16 i.v. (6h)
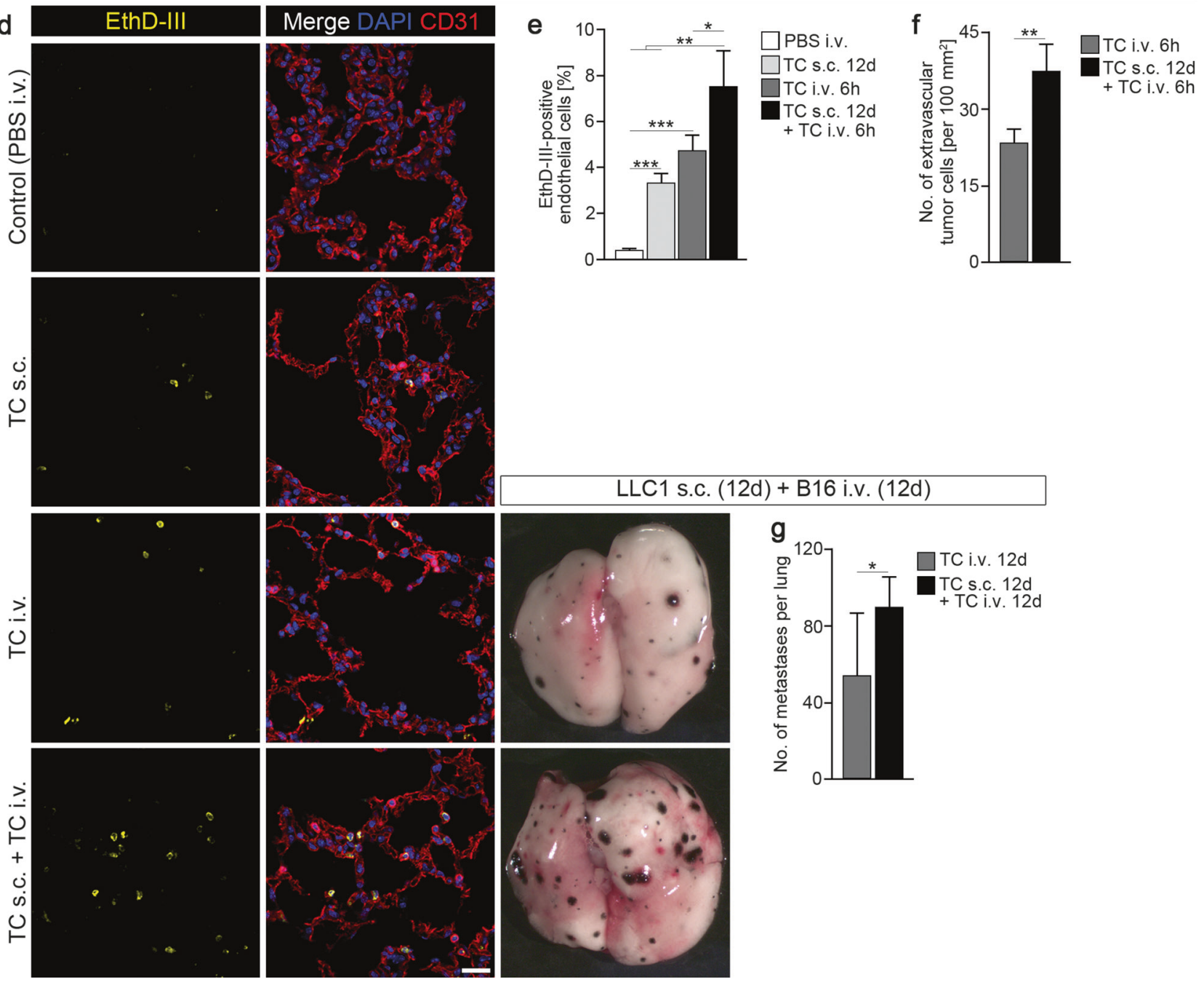
Fig. 4 Primary tumors affect TAK1 and RIPK3 expression in endothelial cells at premetastatic sites and promote endothelial cell necroptosis, tumor cell extravasation, and metastasis. a, b Analysis of MAP3K7 (TAK1) and RIPK3 expression levels by qRT-PCR (a) and quantification of endothelial necroptosis (b) in HUVECs cultured in the presence of MDA-MB-231 TC-conditioned medium. c qRT-PCR analysis of Map3k7 (TAK1) and Ripk3 expression levels in mouse lung endothelial cells (MLECs) isolated from C57B1/6 wild-type mice treated with PBS or harboring subcutaneously implanted B16or LLC1-derived tumors. Relative mRNA expression levels were normalized to GAPDH and to the level detected in control samples. d-g C57Bl/6 wild-type mice were treated with PBS i.v. for $6 \mathrm{~h}$ (control) or with B16 TCs by s.c. injection for $12 \mathrm{~d}$ (TC s.c.), i.v. injection for $6 \mathrm{~h}$ (TC i.v.) or a combination of both (TC s.c. $+\mathrm{TC}$ i.v.) and analyzed for pulmonary EthD-III-positive ECs (e) or extravasated TCs (f). Confocal images of lung sections are shown in (d, left panel). To determine lung metastases, LLC1 primary tumors were subcutaneously implanted for $12 \mathrm{~d}$ followed by B16 i.v. injection and primary tumor excision $24 \mathrm{~h}$ thereafter. Lungs were analyzed another $11 \mathrm{~d}$ later $(\mathbf{g})$. Images of whole lungs are shown in (d, right panel). Shown are representative data of two independent experiments with mean values \pm s.e.m. $(\mathbf{b}, \mathbf{e})$ or \pm s.d. $(\mathbf{a}, \mathbf{c}, \mathbf{f}, \mathbf{g})$ from biological triplicates $(n=3)(\mathbf{a}, \mathbf{b})$ or from $n=4-6$ animals per condition $(\mathbf{c}, \mathbf{e}-\mathbf{g}) .{ }^{*} P<0.05 ; * * P<0.01 ; * * * P<0.001$. Unpaired, two-tailed Student's $t$ test $(\mathbf{a}, \mathbf{b}, \mathbf{f}, \mathbf{g})$ or one-way ANOVA and Bonferroni's post hoc test $(\mathbf{c}, \mathbf{e})$

However, even though the prosurvival function of TAK1 in different cell types is well established, it is not fully understood how loss of TAK1 results in the rerouting of signaling pathways from prosurvival toward cell death. Different factors including the initial stimuli, the cell type and its cellular context seem to be involved [11, 35-39]. In most cases, TAK1 activation engages NF- $\kappa \mathrm{B}$ signaling pathways to transcriptionally regulate genes involved in preventing cell death $[40,41]$. It has also been reported that TAK1 regulates cell survival not solely through NF- $\kappa$ B but also through NF- $\kappa \mathrm{B}$-independent pathways by regulating for example oxidative stress [39] and by regulating members of the RIPK protein family [42]. However, there are conflicting data on the role of RIPK proteins in the context of cell death in TAK1-deficient cells [11, 16, 37, 43, 44]. Our data show that absence of TAK1 in ECs increased RIPK3 expression and that overexpression of RIPK3 resulted in EC necroptosis, which is consistent with data showing that overexpression of necroptosis-related genes is sufficient to promote necroptosis [45-47]. This suggests that sustained TAK1 signaling is important to retain low levels of pronecroptotic molecules in endothelial cells.

In summary, we show that TAK1 in endothelial cells acts to keep necroptosis-promoting molecules (e.g., RIPK3) in check. In situations where TAK1 expression is decreased, for example in tumor-bearing animals, increased endothelial necroptosis facilitates TC extravasation and metastasis. Future work will have to further explore the mechanisms by which primary tumors regulate endothelial necroptosis already before metastatic spreading and whether this might be a basis for novel strategies to prevent or treat metastasis.
Acknowledgments The authors wish to thank Dagmar Magalei and Martina Finkbeiner for their expert technical assistance and Svea Hümmer for excellent secretarial help. This work was supported by the German Cancer Aid and the Max Planck Society.

Author contribution LY performed most of the in vitro and in vivo experiments and analyzed and discussed data; SJ contributed to in vitro and in vivo experiments; TS, JH, and ST contributed to in vitro experiments; SO initiated and supervised the study, discussed data, and commented on the manuscript; BS initiated and supervised the study, performed in vitro and in vivo experiments, discussed data, and wrote the manuscript.

\section{Compliance with ethical standards}

Conflict of interest The authors declare that they have no conflict of interest.

Publisher's note: Springer Nature remains neutral with regard to jurisdictional claims in published maps and institutional affiliations.

\section{References}

1. Reymond N, d'Agua BB, Ridley AJ. Crossing the endothelial barrier during metastasis. Nat Rev Cancer. 2013;13:858-70.

2. Miles FL, Pruitt FL, van Golen KL, Cooper CR. Stepping out of the flow: capillary extravasation in cancer metastasis. Clin Exp Metastasis. 2008;25:305-24.

3. Strilic B, Offermanns S. Intravascular survival and extravasation of tumor cells. Cancer Cell. 2017;32:282-93.

4. Schumacher D, Strilic B, Sivaraj KK, Wettschureck N, Offermanns S. Platelet-derived nucleotides promote tumor-cell transendothelial migration and metastasis via $\mathrm{P} 2 \mathrm{Y} 2$ receptor. Cancer Cell. 2013;24:130-7.

5. Tichet M, Prod'Homme V, Fenouille N, Ambrosetti D, Mallavialle A, Cerezo M, et al. Tumour-derived SPARC drives vascular permeability and extravasation through endothelial VCAM1 signalling to promote metastasis. Nat Commun. 2015;6:6993.

6. Huang RL, Teo Z, Chong HC, Zhu P, Tan MJ, Tan CK, et al. ANGPTL4 modulates vascular junction integrity by integrin signaling and disruption of intercellular VE-cadherin and claudin-5 clusters. Blood. 2011;118:3990-4002.

7. Wolf MJ, Hoos A, Bauer J, Boettcher S, Knust M, Weber A, et al. Endothelial CCR2 signaling induced by colon carcinoma cells enables extravasation via the JAK2-Stat5 and p38MAPK pathway. Cancer Cell. 2012;22:91-105.

8. Strilic B, Yang L, Albarran-Juarez J, Wachsmuth L, Han K, Muller UC, et al. Tumour-cell-induced endothelial cell necroptosis via death receptor 6 promotes metastasis. Nature. 2016;536:215-8.

9. Pasparakis M, Vandenabeele P. Necroptosis and its role in inflammation. Nature. 2015;517:311-20.

10. Shan B, Pan H, Najafov A, Yuan J. Necroptosis in development and diseases. Genes Dev. 2018;32:327-40.

11. Guo X, Yin H, Chen Y, Li L, Li J, Liu Q. TAK1 regulates caspase 8 activation and necroptotic signaling via multiple cell death checkpoints. Cell Death Dis. 2016;7:e2381.

12. Malireddi RKS, Gurung P, Mavuluri J, Dasari TK, Klco JM, Chi $\mathrm{H}$, et al. TAK1 restricts spontaneous NLRP3 activation and cell death to control myeloid proliferation. J Exp Med. 2018;215:1023-34.

13. Sato S, Sanjo H, Takeda K, Ninomiya-Tsuji J, Yamamoto M, Kawai T, et al. Essential function for the kinase TAK1 in innate and adaptive immune responses. Nat Immunol. 2005;6:1087. 
14. Korhonen H, Fisslthaler B, Moers A, Wirth A, Habermehl D, Wieland T, et al. Anaphylactic shock depends on endothelial G (q)/G(11). J Exp Med. 2009;206:411-20.

15. Sivaraj KK, Takefuji M, Schmidt I, Adams RH, Offermanns S, Wettschureck N. G13 controls angiogenesis through regulation of VEGFR-2 expression. Dev Cell. 2013;25:427-34.

16. Mihaly SR, Ninomiya-Tsuji J, Morioka S. TAK1 control of cell death. Cell Death Differ. 2014;21:1667-76.

17. Bertrand MJ, Milutinovic S, Dickson KM, Ho WC, Boudreault A, Durkin J, et al. cIAP1 and cIAP2 facilitate cancer cell survival by functioning as E3 ligases that promote RIP1 ubiquitination. Mol Cell. 2008;30:689-700.

18. Feoktistova M, Geserick P, Kellert B, Dimitrova DP, Langlais C, Hupe M, et al. cIAPs block Ripoptosome formation, a RIP1/ caspase- 8 containing intracellular cell death complex differentially regulated by cFLIP isoforms. Mol Cell. 2011;43:449-63.

19. Peinado H, Zhang H, Matei IR, Costa-Silva B, Hoshino A, Rodrigues G, et al. Pre-metastatic niches: organ-specific homes for metastases. Nat Rev Cancer. 2017;17:302-17.

20. Storey BT, Pittman HK, Christian JF, Haisch CE, Verbanac KM. Characterization of Lewis lung clonal variants in a model of syngeneic pulmonary murine metastases. Clin Exp Metastasis. 2004;21:265-73.

21. Hiratsuka S, Nakamura K, Iwai S, Murakami M, Itoh T, Kijima H, et al. MMP9 induction by vascular endothelial growth factor receptor-1 is involved in lung-specific metastasis. Cancer Cell. 2002;2:289-300.

22. Lin YC, Chang LY, Huang CT, Peng HM, Dutta A, Chen TC, et al. Effector/memory but not naive regulatory $\mathrm{T}$ cells are responsible for the loss of concomitant tumor immunity. J Immunol. 2009;182:6095-104.

23. Turk MJ, Guevara-Patino JA, Rizzuto GA, Engelhorn ME, Sakaguchi S, Houghton AN. Concomitant tumor immunity to a poorly immunogenic melanoma is prevented by regulatory $\mathrm{T}$ cells. J Exp Med. 2004;200:771-82.

24. Liu Y, Cao X. Characteristics and significance of the premetastatic niche. Cancer Cell. 2016;30:668-81.

25. Chen Y, Zhou B, Xu L, Fan H, Xie J, Wang D. MicroRNA-146a promotes gastric cancer cell apoptosis by targeting transforming growth factor beta-activated kinase 1. Mol Med Rep. 2017;16:755-63.

26. Huang FT, Peng JF, Cheng WJ, Zhuang YY, Wang LY, Li CQ, et al. MiR-143 targeting TAK1 attenuates pancreatic ductal adenocarcinoma progression via MAPK and NF-kappaB pathway in vitro. Dig Dis Sci. 2017;62:944-57.

27. Yuan G, Zhao Y, Wu D, Gao C, Jiao Z. miRNA-20a upregulates TAK1 and increases proliferation in osteosarcoma cells. Future Oncol. 2018;14:461-9.

28. Zhao N, Wang R, Zhou L, Zhu Y, Gong J, Zhuang SM. MicroRNA-26b suppresses the NF-kappaB signaling and enhances the chemosensitivity of hepatocellular carcinoma cells by targeting TAK1 and TAB3. Mol Cancer. 2014;13:35.

29. Su Z, Yang Z, Xu Y, Chen Y, Yu Q. MicroRNAs in apoptosis, autophagy and necroptosis. Oncotarget. 2015;6:8474-90.

30. Zhou W, Fong MY, Min Y, Somlo G, Liu L, Palomares MR, et al. Cancer-secreted miR-105 destroys vascular endothelial barriers to promote metastasis. Cancer Cell. 2014;25:501-15.
31. Qiu Z, Li H, Wang J, Sun C. miR-146a and miR-146b in the diagnosis and prognosis of papillary thyroid carcinoma. Oncol Rep. 2017;38:2735-40.

32. Yang R, Fu Y, Zeng Y, Xiang M, Yin Y, Li L, et al. Serum miR$20 \mathrm{a}$ is a promising biomarker for gastric cancer. Biomed Rep. 2017;6:429-34.

33. Huang Y, Song N, Ding Y, Yuan S, Li X, Cai H, et al. Pulmonary vascular destabilization in the premetastatic phase facilitates lung metastasis. Cancer Res. 2009;69:7529-37.

34. Hiratsuka S, Watanabe A, Aburatani H, Maru Y. Tumourmediated upregulation of chemoattractants and recruitment of myeloid cells predetermines lung metastasis. Nat Cell Biol. 2006;8:1369-75.

35. Simmons AN, Kajino-Sakamoto R, Ninomiya-Tsuji J. TAK1 regulates Paneth cell integrity partly through blocking necroptosis. Cell Death Dis. 2016;7:e2196.

36. Lamothe B, Lai Y, Xie M, Schneider MD, Darnay BG. TAK1 is essential for osteoclast differentiation and is an important modulator of cell death by apoptosis and necroptosis. Mol Cell Biol. 2013;33:582-95.

37. Morioka S, Broglie P, Omori E, Ikeda Y, Takaesu G, Matsumoto $\mathrm{K}$, et al. TAK1 kinase switches cell fate from apoptosis to necrosis following TNF stimulation. J Cell Biol. 2014;204:607-23.

38. Morioka S, Inagaki M, Komatsu Y, Mishina Y, Matsumoto K, Ninomiya-Tsuji J. TAK1 kinase signaling regulates embryonic angiogenesis by modulating endothelial cell survival and migration. Blood. 2012;120:3846-57.

39. Omori E, Morioka S, Matsumoto K, Ninomiya-Tsuji J. TAK1 regulates reactive oxygen species and cell death in keratinocytes, which is essential for skin integrity. J Biol Chem. 2008;283:26161-8.

40. Hayden MS, Ghosh S. Shared principles in NF-kappaB signaling. Cell . 2008;132:344-62.

41. Sakurai H. Targeting of TAK1 in inflammatory disorders and cancer. Trends Pharmacol Sci. 2012;33:522-30.

42. Geng J, Ito Y, Shi L, Amin P, Chu J, Ouchida AT, et al. Regulation of RIPK1 activation by TAK1-mediated phosphorylation dictates apoptosis and necroptosis. Nat Commun. 2017;8:359.

43. Vanlangenakker $\mathrm{N}$, Vanden Berghe $\mathrm{T}$, Bogaert $\mathrm{P}$, Laukens $\mathrm{B}$, Zobel K, Deshayes K, et al. cIAP1 and TAK1 protect cells from TNF-induced necrosis by preventing RIP1/RIP3-dependent reactive oxygen species production. Cell Death Differ. 2011;18:656-65.

44. Dondelinger Y, Aguileta MA, Goossens V, Dubuisson C, Grootjans S, Dejardin E, et al. RIPK3 contributes to TNFR1mediated RIPK1 kinase-dependent apoptosis in conditions of cIAP1/2 depletion or TAK1 kinase inhibition. Cell Death Differ. 2013;20:1381-92.

45. Zhang T, Zhang Y, Cui M, Jin L, Wang Y, Lv F, et al. CaMKII is a RIP3 substrate mediating ischemia- and oxidative stress-induced myocardial necroptosis. Nat Med. 2016;22:175-82.

46. Zhang DW, Shao J, Lin J, Zhang N, Lu BJ, Lin SC, et al. RIP3, an energy metabolism regulator that switches TNF-induced cell death from apoptosis to necrosis. Science. 2009;325:332-6.

47. Gautheron J, Vucur M, Schneider AT, Severi I, Roderburg C, Roy $\mathrm{S}$, et al. The necroptosis-inducing kinase RIPK3 dampens adipose tissue inflammation and glucose intolerance. Nat Commun. 2016;7:11869. 